\title{
Experimental demonstration of optical phase conjugation using counter-propagating dual pumped four-wave mixing in semiconductor optical amplifier
}

\author{
Abhishek Anchal ${ }^{a}$, Pradeep Kumar $\mathrm{K}^{\mathrm{b}}$, Sean O'Duill ${ }^{\mathrm{c}}$, Prince M. Anandarajah ${ }^{\mathrm{C}}$, Pascal Landais ${ }^{\mathrm{C}}$ \\ ${ }^{a}$ Department of Electrical Engineering, IIT Kanpur, Kanpur 208016, India \\ ${ }^{\mathrm{b}}$ Center for Lasers and Photonics, IIT Kanpur, Kanpur 208016, India \\ ${ }^{c}$ School of Electronic Engineering, Dublin City University, Glasnevin, Dublin 9, Ireland
}

\begin{abstract}
We report optical phase conjugation in C-band by counter-propagating dual pumped non-degenerate fourwave mixing in a semiconductor optical amplifier (SOA). The co-propagating signal and pump waves create a grating inside SOA which diffracts counter-propagating pump and generates the conjugate wave. Since the signal and conjugate waves appear at opposite ends, the conjugate is easily filtered out from the rest of spectrum with minimal spectral shift of the conjugate with respect to the incoming signal. With pump powers of $3.2 \mathrm{dBm}$ each and signal input power of $7 \mathrm{dBm}$, conjugate power was of $27.2 \mathrm{dBm}$, giving a conversion efficiency of $1 \%$ at $18 \mathrm{GHz}$ pump-signal detuning. By modulating the signal by a periodic pattern '1000' at 10 Gbps using a non-zero chirp intensity modulator and resolving the temporal profile of the electric field envelope of the conjugate wave, we demonstrate spectral in-version.
\end{abstract}

\section{Introduction}

Optical phase conjugation (OPC) is used in several applications such as spectroscopy, interferometry, optical squeezing, and op-tical data processing (see [1] and references therein). In the con-text of optical fiber communications, OPC is used to mitigate fiber dispersion and nonlinearities by mid-span spectral inversion in which impairments due to fiber dispersion and nonlinearities in the first half of the fiber are inverted by OPC, and compensated by subsequent transmission through the second half of the fiber [2-5].

OPC can be generated in many ways; three-wave mixing in periodically poled LiNbO3, four-wave mixing in fibers and SOAs, and backward stimulated Raman and Brillouin scattering in fibers $[1,2,6]$. In $[7,8]$, a bidirectional pumping scheme in a SOA to generate OPC was theoretically proposed and the appearance of a signal and conjugate at opposite ends of the SOA was predicted. In [9], a bidirectionally pumped OPC scheme was demonstrated in a SOA using orthogonally polarized counter-propagating pumps of the same wavelength. The orthogonally polarized pumps were used to avoid the reflection of the signal at input port due to the formation of Bragg grating inside SOA, which can lead to difficulty in filtering of conjugate. The signal and conjugate waves appeared at opposite ends of the SOA, also orthogonally polarized. A low conversion efficiency of $0.2 \%$ was obtained.

In this paper, we generate OPC in an SOA using counter-propagating dual pumped FWM. Pump1 and signal waves are injected into one end of the SOA and a second pump is injected into the other end. The beating between pump1 and signal waves creates a refractive index and gain gratings; the pump2 senses this grating and scatters, to generate conjugated copy of original signal wave. The conjugate and signal waves appear at 
opposite ends of the SOA; the conjugate wave is filtered out using an optical filter. With pump and injected signal powers of $-3.2 \mathrm{dBm}$ and $-7 \mathrm{dBm}$ respectively, the conjugate power was $-27.2 \mathrm{dBm}$ giving the higher recorded conversion efficiency of $1 \%$ up to $12 \mathrm{GHz}$ pump1-signal detuning [9]. By modulating the signal wave with a non-zero chirp intensity modulator and resolving the temporal profile of the electric field envelope of the conjugate wave, we demonstrate spectral inversion.

The rest of the paper is organized as follows. In Section 2, we briefly discuss the theory of counter-propagating dual pumped FWM, leading to OPC generation in SOA. The spectrum at the output of SOA shows the generation of desired conjugate as well as additional FWM products. We measure the efficiency of conjugate generation as a function of pump-signal frequency detuning and compare it to theoretical values. The temporal resolution of signal and conjugate waves indicates phase inversion. In Section 3, we discuss our experimental setup and results of OPC generation. The spectrum at the output of SOA shows the generation of desired conjugate as well as additional FWM products. We measure the efficiency of conjugate generation as a function of pump-signal frequency detuning and compare it to theoretical values. The temporal resolution of signal and conjugate waves indicates phase inversion. Finally, in Section 4, we conclude by summarizing our results.

\section{Theory}

Fig. 1 shows the schematic diagram of counter-propagating dual-pumped FWM in SOA. The wavelengths $\lambda_{i}$ and frequencies $\omega_{i}, i=1,2,3,4$ correspond to pump $p_{1}$, pump $p_{2}$, signal and conjugate waves respectively. The pump1 and signal waves are injected into one end of the SOA and pump 2 is injected into the other end. Beating between pump $p_{1}$ and signal creates a complex-valued grating - real part corresponds to gain grating and imaginary part corresponds to index grating - in the active layer of the SOA. The origin of the gratings is found in the carrier density pulsation able to sustain modulation at $300 \mathrm{GHz}$ well above its cut-off frequency [7]. The grating diffracts pump $p_{2}$ to create two sidebands at $\omega_{2}-\Omega$ and $\omega_{2}+\Omega$, where $\Omega=\omega_{1}-\omega_{3}$ is the detuning. The sideband $\omega_{2}+\Omega$ corresponds to wavelength $\lambda_{4}$, which is conjugated version of the signal and propagates in the direction opposite to signal. The other sideband $\omega_{2}-\Omega$ is the non-conjugate version of signal, denoted by idler3. Conjugate and non-conjugate copies of signal along with the amplified pump $p_{2}$ is present at port 1 as shown in box labelled $\mathbf{A}$ in Fig. 1. A similar interaction between pump $\mathbf{p}_{2}$ and signal waves creates an additional weak grating and is neglected. Other possible FWM interaction is between pump1 and signal, which results in generation of idler 2 which is present along with amplified pump1 and signal at port 2 shown in the box B of Fig. 1. We summarize different FWM product in Table 1.

The equations governing temporal evolution of signal and generation of conjugate wave can be obtained by substituting the total electric field $E=\sum_{i}^{4}=1 A_{i}(z, t) \exp \left(j\left(\omega_{i} t+(-1)^{i} k_{i} z\right)\right)$ into the nonlinear Schrödinger equation [10] :

$$
\nabla^{2} E-\frac{n^{2}}{c^{2}} \frac{\partial^{2} E}{\partial t^{2}}=\frac{1}{\epsilon_{0} c^{2}} \frac{\partial^{2} P_{N L}}{\partial t^{2}},
$$

where PNL denotes the induced non-linear polarization. For simplicity of analytical solution we neglected the contribution of idler2 and idler3. Using SOA-FWM analysis outlined in [10], the propagation equation for the forwards and backwards travelling conjugate waves are assuming that the gain saturation is dominated by the pumps: 


$$
\begin{aligned}
& \frac{\partial A_{3}}{\partial z}=-\alpha_{z} \frac{\partial A_{3}}{\partial z}=-\alpha_{3} A_{3}+j_{\kappa_{3}} A_{4}^{*} \exp (j \Delta k z) \\
& \frac{\partial A_{4}^{*}}{\partial z}=\alpha_{4}^{*} A^{\frac{\partial A_{4}^{*}}{\partial z}=\alpha_{4}^{*} A_{4}^{*}+j \kappa_{4}^{*} A_{3} \exp (-j \Delta k z)}
\end{aligned}
$$

$\alpha_{4}, K_{3}$, and $k_{4}$ are given in [10]. These equations are valid for small pump-signal detuning. In our experiments we initially keep the pump-signal detuning to within $10 \mathrm{GHz}$; The detuning is varied up to $40 \mathrm{GHz}$ to study its effect on FWM conversion efficiency. Solving subject to boundary conditions, $A_{3}(0)=A_{30}$ and $A_{4}(L)=0$, we obtain

$$
\begin{aligned}
& A_{3}(z)=A_{30} e^{\left(\mu-\alpha_{3}\right) z}\left[\cos \zeta z+\frac{\zeta \sin \zeta L-\mu \cos \zeta L}{\mu \sin \zeta L+\zeta \cos \zeta L} \sin \zeta z\right] \\
& A_{4}(z)=-j \kappa_{4} A_{30}^{*} e^{-\left(\mu^{*}-\alpha_{4}\right) z}\left[\frac{\sin \zeta(L+z)}{\mu \sin \zeta L+\zeta \cos \zeta L}\right]^{*}
\end{aligned}
$$

where $\mu=\left(\alpha_{3}+\alpha_{4}{ }^{*}+\Delta k\right) / 2$ and $\zeta=\operatorname{sqrt}\left((2 \mu)^{2}-\kappa_{3} k_{4}\right) / 2$. This shows that $A_{4}(z)$ is conjugate of $A_{30}$. The boundary condition for the backwards travelling conjugate is $A_{4}(z=L)=0$; therefore this back-ward travelling idler builds up from the right-hand side of (3) which is proportional to the complex conjugate of the input signal A3. We define conversion efficiency as ratio of output conjugate power at $z=0$ to input signal power at $\mathrm{z}=0$

$$
\eta=\frac{\left|A_{4}(0)\right|^{2}}{\left|A_{3}(0)\right|^{2}}
$$

Putting the value of $|A 3(0)|$ and $|A 4(0)|$ from (3)

We observe that the conversion efficiency $\eta$ depends upon detuning $\Omega$ through $\mathrm{k}$ in $\mu$ and $\zeta$. The expression of efficiency $\eta$ looks similar to the reflectivity $R$ in [10], but their values differ in magnitude as the parameters are different in the two expressions.

\section{Experimental setup and results}

\subsection{Setup}

Fig. 2 shows our experimental setup to generate phase con-jugated wave. The setup consists of an SOA (CIP SOA-XN-OEC-1550) as nonlinear medium to realize FWM. The small signal gain of the SOA is $34 \mathrm{~dB}$ with $<1 \mathrm{~dB}$ polarization dependence. The CW optical signal from a tunable laser (TL1) at $\lambda 3=1558.08 \mathrm{~nm}$ is fed to port 1 of the SOA along with a pump1 $(\lambda 1=1557.89 \mathrm{~nm})$ from other tunable laser (TL3) through a $3 \mathrm{~dB}$ coupler and a circulator CIRC1. Another tunable laser TL2 $(\lambda 2=1559.01 \mathrm{~nm})$, acting as pump2, is fed to port 2 of the SOA through a circulator CIRC2. A polarization controller PC1 was used to align the polarization of the signal wave to maximize transmission through Mach-Zehnder modulator (MZM). PC2 was used to align the polarization of pump1 and signal waves to achieve maximum FWM efficiency, whereas pump2 can be of any arbitrary polarization. A part of signal is reflected, when the two pumps are off, thereby confirming that signal is reflected due to end facet reflectivity of SOA rather than due to Bragg grating [9]. An optical tunable filter (OTF), Yenista XTM-50 with a variable bandwidth set to $0.2 \mathrm{~nm}$ and sharp roll-off greater than $100 \mathrm{~dB} / \mathrm{nm}$ was used to isolate the conjugate. The optical spectrum was measured at the circulator ports I and II as well as the port 1 and 2 of the SOA using complex optical spectrum analyzer OSA1,2 (APEX AP2443B). 
In order to observe phase conjugation, we introduce chirping by modulating the signal with the aid of an MZM, driven with the repetitive bit pattern of ' 1000 ' at a bit rate of $10 \mathrm{Gbps}$. The repetition of the 4 bit pattern creates harmonics at multiples of $2.5 \mathrm{GHz}$, which is required by the complex OSA for spectral ana-lysis [11]. MZM used is z-cut fixed-chirp intensity modulator, in which optical or RF power unbalancing results in the chirping. The optical spectrum at port I and port II of the circulators, CIRC1 and CIRC2, at the two ends of the SOA, as well as the chirping of input signal and conjugate was measured using a complex OSA. The complex OSA was synchronized to the data pattern by feeding it with a $10 \mathrm{GHz}$ clock from the pattern generator.

In our experiments, the SOA is driven at $400 \mathrm{~mA}$ bias current. Pump ${ }_{1}$ (and Pump $\mathrm{P}_{2}$ ) and signal power are kept at $-3.2 \mathrm{dBm}$ and $-7 \mathrm{dBm}$ respectively. Detuning between pump1 and signal is kept small $(0.2 \mathrm{~nm})$ to achieve a high conjugate power.

\subsection{Results and discussion}

Fig. 3a shows the spectrum of the FWM products with the modulated input signal with ' 1000 ' pattern at 10 Gbps as ex-plained earlier at port 1 of SOA. Sidebands about signal frequencies appear at spacing of $2.5 \mathrm{GHz}$. Fig. $3 \mathrm{~b}$ shows the spectrum of the conjugate after filtering. Spectrum of the backward travelling conjugate wave is reversed to that of input signal, indicating phase conjugation. Idler3 is backward travelling but nonconjugate copy of the signal. These conjugate and non-conjugate copies of signal along with amplified pump2 is obtained at port 1 as shown in the box A, as described in Fig. 1. Idler 2 is also present at port 1 together with pump1 and signal due to reflection from the end facet of SOA as shown in the box B of Fig. 3. We see that sideband is present in pump 1 , pump 2 , idler, and idler ${ }_{3}$. This is due to the generation of additional FWM products by the interaction of sidebands of signal with pumps.

The signal power input from the TL1 was kept at $-7 \mathrm{dBm}$. The output conjugate power was measured to be $27.2 \mathrm{dBm}$, thus giving an overall conversion efficiency $\eta$ of $1 \%$. The SOA used in our experiment is driven at high bias current at $400 \mathrm{~mA}$ due to long active region, resulted in the five times higher efficiency than the previously reported value in [9].

Fig. 3 c shows measured conversion efficiency $\eta$, of the back-ward travelling conjugate as a function of detuning between the signal and pump1. We compared the experimental value of conversion efficiency with the theoretical value obtained from (4). We observed that the conjugate efficiency decreases with detuning in both experiment and theory, with $3 \mathrm{~dB}$ bandwidth of $12 \mathrm{GHz}$ and $9 \mathrm{GHz}$ respectively. However, in theoretical prediction of efficiency obtained from (4), we considered only the backward travelling conjugate and neglected the other FWM products, which was present in the experimental spectrum according to Fig. 3a. This results in the smaller experimental value of efficiency than that of theoretical prediction given in (4).

Fig. 4 shows plot of temporal profile of intensity and phase of signal and conjugate waves versus time as displayed by the OSA. Dashed line in Fig. 4a shows intensity modulation of signal at 10 Gbps by MZM and solid line shows the chirping introduced in signal by modulator. The ' 1000 ' intensity modulation pattern is clearly visible along with the phase variation shown by the solid line. A noticeable amount of phase change occurs during the bit transition from the lower to the upper level. Fig. $4 \mathrm{~b}$ displays a similar intensity variation of the conjugate wave shows modulation information transfer from signal. We also observe phase inversion in the conjugate wave, though there is spurious phase variation in conjugate waves, which results in approximate phase inversion in signal. This approximate phase inversion is further support by comparing the phase slope ( $1 /(2 \pi) \partial \varphi / \partial t)$ of signal and conjugate waves at $A B$ and $C D$ with $A B^{\prime \prime}$ and $C^{\prime} D^{\prime}$, which are $24 \mathrm{GHz}$ and $25 \mathrm{GHz}$ respectively. The spurious phase variation in conjugate wave is possibly due to the cross gain modulation on the pump that interferes with the conjugate and non-linear dependence of conversion efficiency on detuning, which causes the extra oscillations in the phase waveform or small signal and conjugate power results in small peak-to-peak chirp. By introducing large chirp in signal, we can neglect the small oscillation in phase and a clear phase inversion is observed. 


\section{Conclusion}

In summary, we have shown experimentally that dual counter-propagating pumped FWM in SOA can be used to generate optical phase conjugation with signal and conjugate appearing at opposite ends of the SOA. This spatial separation between signal and conjugate facilitates the filtering of the conjugate and allows for the reduction of the spectral separation between the signal and conjugate waves. We observe that the reflected signal is present even when the two pumps are turned off, confirms that the signal is reflected due to end facet reflectivity of SOA. This restricts us to achieve phase conjugation at the signal wavelength. In principle by using SOA with small end facet reflectivity $\left(<10^{-4}\right)$ and placing pump wavelengths symmetrically around signal, wavelength-shift free phase conjugation can be achieved.

Phase conjugation was obtained with an overall FWM efficiency of $1 \%$ at $18 \mathrm{GHz}$ of pump1-signal detuning. Time resolved measurement of electric field using complex OSA shows that phase of conjugate wave is approximately inverted with respect to signal phase. Cross gain modulation and residual chirp present in signal and conjugate, restricts the exact inversion of phase in conjugate, which can be avoided by increasing chirp in the signal.

\section{Acknowledgments}

Funding: This research is supported by a Marie Curie International Research Staff Exchange Scheme Fellowship within the 7th European community framework programme (Grant agreement number 318941). 


\section{References}

[1] G.S. He, Optical phase conjugation: principles, techniques and applications, Prog. Quantum Electron. 26 (2002) 131-191.

[2] S.L. Jansen, D. van den Borne, P.M. Krummrich, S. Spalter, G.-D. Khoe, H. de Waardt, Long-haul DWDM transmission systems employing optical phase conjugation, IEEE J. Sel. Top. Quantum Electron. 12 (2006) 505520.

[3] M. Morshed, L.B. Du, B. Foo, M.D. Pelusi, B. Corcoran, A.J. Lowery, Experimental demonstrations of dual polarization CO-OFDM using mid-span spectral in-version for nonlinearity compensation, Opt. Exp. 22 (2014) 10455-10466.

[4] X. Xiang-Jun, Ma Jian-Xin, Z. Qi, D. Chao-Gong, W. Kui-Ru, Y. Chong-Xiu, L. Bo, $155 \mathrm{Mb} / \mathrm{s}$ and $10 \mathrm{~Gb} / \mathrm{s}$ combined FSK-IM/optical label-packet modulation signals $100 \mathrm{~km}$ transmission over standard single mode fiber using mid-span spectral inversion by four-wave mixing in an SOA, Chin. Phys. B 18 (2009) 3449-3452.

[5] Xiang Liu, A.R. Chraplyvy, P.J. Winzer, R.W. Tkach, S. Chandrasekhar, Phase-conjugated twin waves for communication beyond the Kerr nonlinearity limit, Nat. Photon. 7 (2013) 560-568.

[6] M.F.C. Stephens, M. Tan, I.D. Phillips, S. Sygletos, P. Harper, N.J. Doran, 1.14 Tb/s DP-QPSK WDM polarization-diverse optical phase conjugation, Opt. Exp. 22 (2014) 11840-11848.

[7] G.P. Agrawal, Population pulsation and nondegenerate four-wave mixing in semiconductor laser and amplifiers, J. Opt. Soc. Am. B 5 (1988) 147-159.

[8] S. Bischoff, A. Buxens, H.N. Poulsen, A.T. Clausen, J. Mørk, Bidirectional four-wave mixing in semiconductor optical amplifiers: theory and experiment, J. Lightwave Technol. 17 (1999) 1617-1625.

[9] C.L. Janer, M.J. Connelly, Optical phase conjugation technique using four-wave mixing in semiconductor optical amplifier, Electron. Lett. 47 (2011).

[10] G.P. Agrawal, Four-wave mixing and phase conjugation in semiconductor laser media, Opt. Lett. 12 (1987) 260-262.

[11] Notes on Optical Complex Spectrum Analyzer: Measurement Principle for APEX AP2441B/AP2443B. 〈http://www.apex-t.com/ocsa-principle//. 


\section{Figures \& Table}

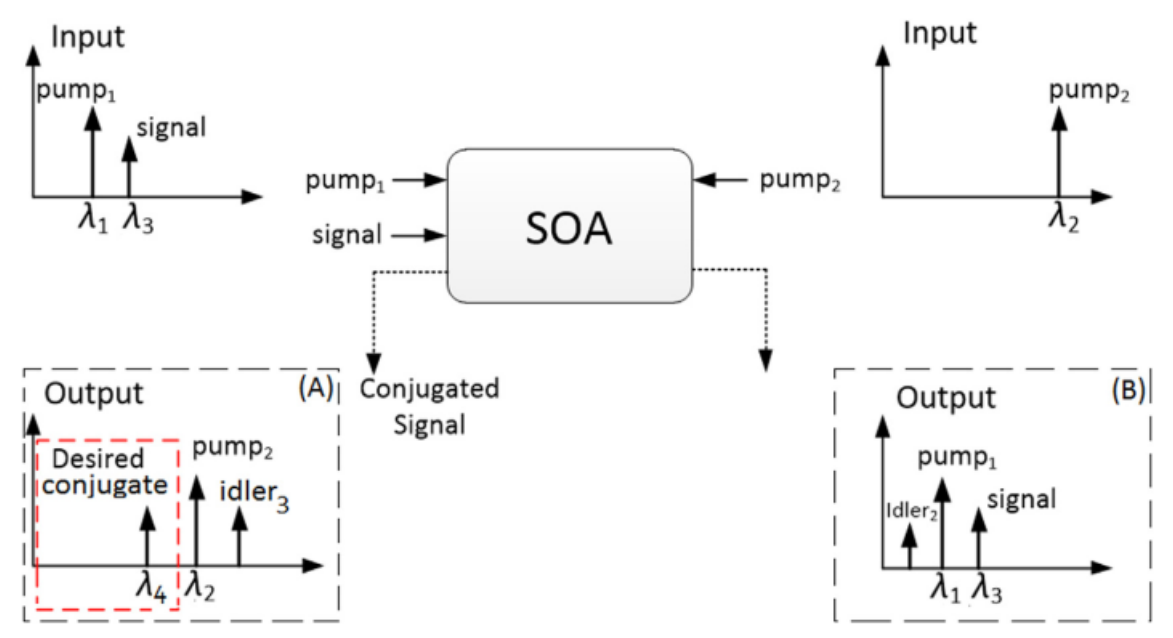

Fig. 1. Block-diagram of dual pumped counter-propagating FWM in SOA. Pumps are detuned from symmetrical position of signal to facilitate optical filtering of conjugate. $\lambda_{1}$ and $\lambda_{2}$ : forward and backward pump wavelength, $\lambda 3$ : signal, and $\lambda 4$ : conjugate wavelength 


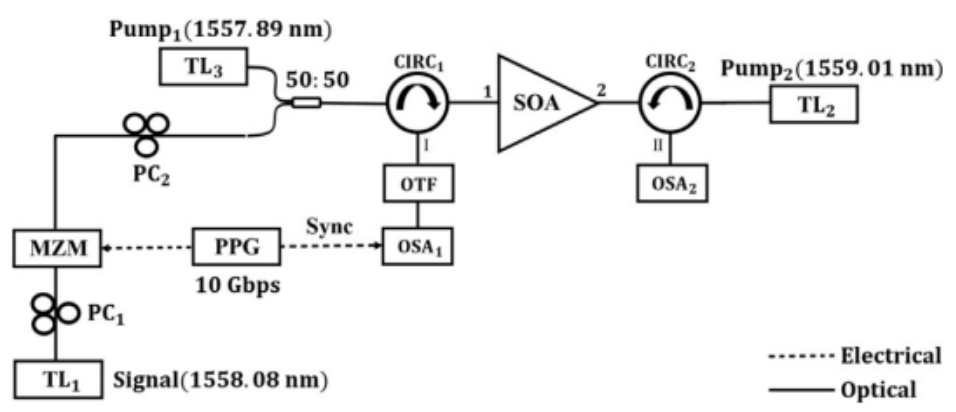

Fig. 2. Generation of phase conjugated wave using counter-propagating dual pumps in SOA. $T L_{1,2,3}$ : tunable laser, MZM: Mach-Zehnder modulator, PPG: pulse pattern generator, $\mathrm{CIRC}_{1,2}$ : circulator, SOA: semiconductor optical amplifier, OTF: optical tunable filter, OSA 1,2 : complex optical spectrum analyzer. 


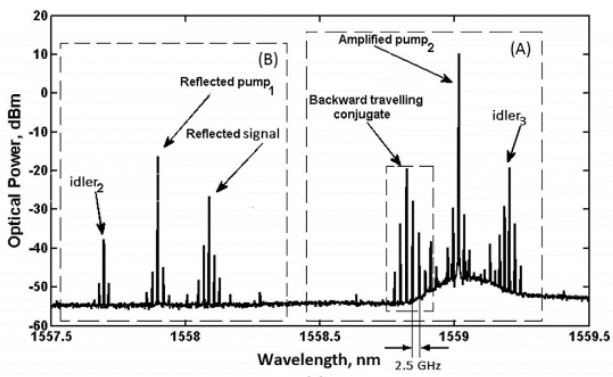

(a)

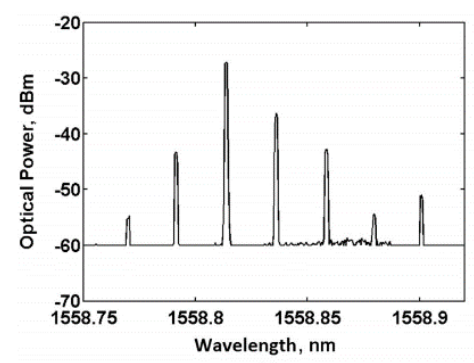

(b)

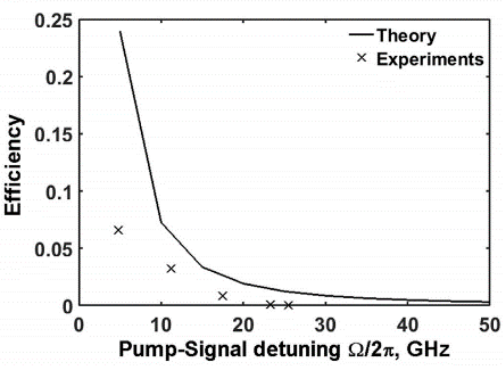

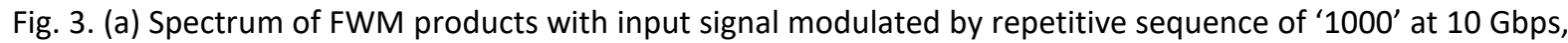
(b) spectrum of backward propagating conjugate after filtering by OTF. Note flipping of spectrum at backward travelling conjugate $(1558.83 \mathrm{~nm}$ ) due to phase conjugation, and (c) dependency of conversion efficiency of backward travelling conjugate with detuning between pump1 and signal. 


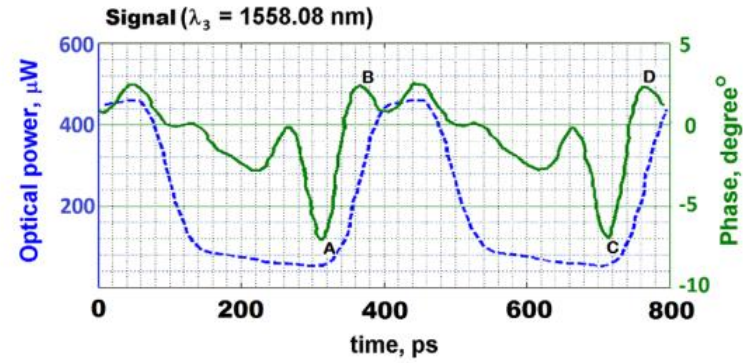

(a)

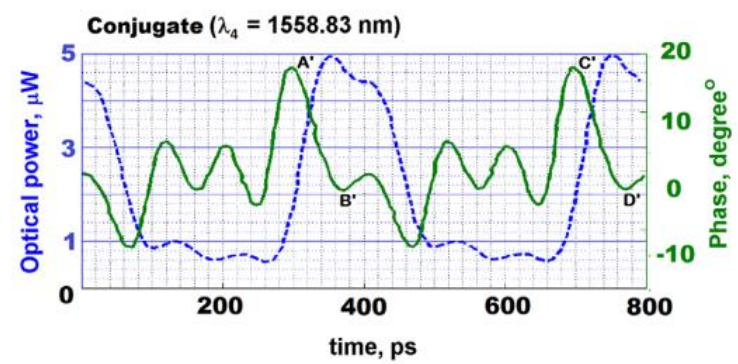

(b)

Fig. 4. Intensity and phase vs time plot of (a) input signal, $\lambda_{3}=1558.08 \mathrm{~nm}$ and (b) filtered output (conjugate), $\lambda_{4}=1558.83 \mathrm{~nm}$. Dashed line - intensity. Solid line - phase. We observe phase inversion at bit transition AB$A B^{\prime \prime}$ and $C D-C^{\prime} D^{\prime}$. 
Table 1

FWM products in dual pumped counter-propagating FWM in SOA

\begin{tabular}{lll}
\hline Interacting waves & FWM product & Comments \\
\hline$\omega_{1}, \omega_{2}$, and $\omega_{3}$ & $\omega_{2}+\omega_{1}-\omega_{3}$ & Conjugate counter-propagating \\
& $\omega_{2}-\omega_{1}+\omega_{3}$ & Non-conjugate counter-propagating \\
$\omega_{1}$ and $\omega_{3}$ & $2 \omega_{1}-\omega_{3}$ & FWM product co-propagating \\
\hline
\end{tabular}

